\title{
Characterization of primary neurospheres generated from mouse ventral rostral hindbrain
}

\author{
Nadja Osterberg • Eleni Roussa
}

Received: 11 November 2008 / Accepted: 10 December 2008 /Published online: 18 February 2009

(C) The Author(s) 2009. This article is published with open access at Springerlink.com

\begin{abstract}
Serotonergic (5-HT) neurons of the reticular formation play a key role in the modulation of behavior, and their dysfunction is associated with severe neurological and psychiatric disorders, such as depression and schizophrenia. However, the molecular mechanisms underlying the differentiation of the progenitor cells and the specification of the 5-HT phenotype are not fully understood. A primary neurosphere cell-culture system from mouse ventral rostral hindbrain at embryonic day 12 was therefore established. The generated primary neurospheres comprised progenitor cells and fully differentiated neurons. Bromodeoxyuridine incorporation experiments in combination with immunocytochemistry for neural markers revealed
\end{abstract}

This work was supported by the Deutsche Forschungsgemeinschaft through the DFG-Research Center for Molecular Physiology of the Brain (CMPB).

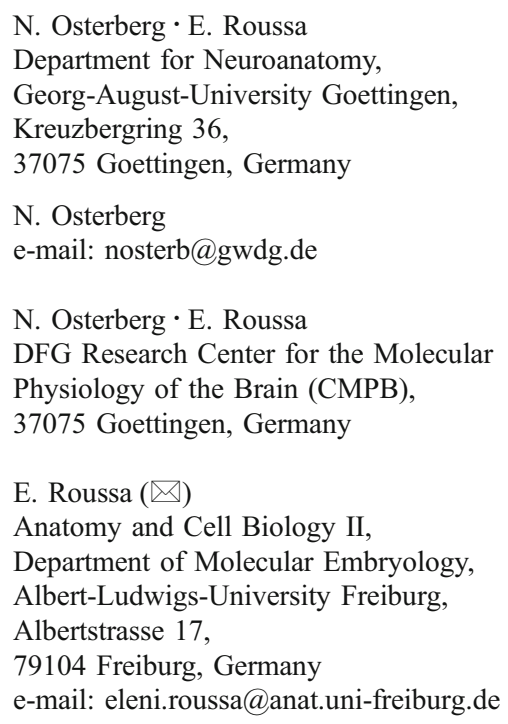

the proliferation capacity of the neural multipotent hindbrain progenitors within neurospheres and their ability to differentiate toward the neuronal lineage and serotonergic phenotype. Gene expression analysis by reverse transcription with the polymerase chain reaction showed that the neurospheres were regionally specified, as reflected by the expression of the transcription factors Gata2 and Pet1. Treatment of dissociated primary neurospheres with exogenous Shh significantly increased the number of 5-HTimmunopositive cells compared with controls, whereas neutralization of endogenous Shh significantly decreased the number of 5-HT neurons. Thus, the primary neurosphere culture system presented here allows the expansion of hindbrain progenitor cells and the experimental control of their differentiation toward the serotonergic phenotype. This culture system is therefore a useful model for in vitro studies dealing with the development of 5-HT neurons.

Keywords Neurogenesis · Serotonergic neurons · Primary neurospheres $\cdot$ Mouse (NMRI)

\section{Introduction}

Serotonin-producing neurons are localized in the midline of the reticular formation within the brainstem of the adult brain. They arise as two distinct clusters, subdivided into nine raphe nuclei (B1-B9) in the hindbrain (Levitt and Moore 1978). The rostral serotonergic (5-HT) groups (B5B9) have ascending projections in various areas of the central nervous system (CNS), such as the hypothalamus, hippocampus, cerebral cortex, basal ganglia, and amygdala, whereas the caudal raphe nuclei (B1-B4) mainly project to the spinal cord. Although 5-HT neurons constitute only a small fraction of the total neuron population of the CNS, 
they are essential for the modulation of behavior such as appetite, sleep-wake cycle, anxiety, memory, and mood. Dysfunction of the 5-HT system is associated with severe neurological and psychiatric disorders as depression, schizophrenia, and migraine (Nelson and Chiavegatto 2001; Zhang et al. 2005). Thus, studies uncovering the molecular mechanisms underlying the differentiation of progenitor cells and the specification of the 5-HT phenotype are of major importance.

According to the prevailing model, the development of 5-HT neurons depends critically on the floor plate signal sonic hedgehog (Shh), on fibroblast growth factor 8 (FGF8) from the midbrain-hindbrain boundary and on the early prepattering signal FGF4 (Ye et al. 1998). In addition, the transcription factors Nkx2.2., Lmx1b, Mash1, Pet1, and Gata2 are apparently necessary for the induction and specification of rostral 5-HT neurons (Hendricks et al. 1999; Pattyn et al. 2003, 2004; Chen and Ding 2006; Zhao et al. 2006). However, the complete transcriptional network regulating the development of 5-HT neurons in the embryonic CNS is still unknown. Moreover, investigation of the complex network of intrinsic and extrinsic factors mediating the development of rostral 5-HT neurons is challenging because of the cellular heterogeneity and the relatively low number of 5-HT neurons within the rostral hindbrain.

For several brain areas, the neurosphere culture system has become a useful tool for the expansion of neural stem and progenitor cells because of their proliferation capacity in response to mitogens. This culture system was originally considered to derive from a single stem cell, but this issue is now a matter of controversy. More recently, the differentiation of neural multipotent progenitors from several brain regions into specific cell types in vitro has been monitored after extensive expansion by multi-passaging of the neurospheres (Reynolds and Weiss 1992; Morshead et al. 1994; Palmer et al. 1997; Gage 2000; Roisen et al. 2001). Such a system is certainly of advantage for the study of certain questions, such as the determination of principal stem cell properties, or for the experimental expansion of cells for therapeutic approaches. However, one consequence of extensive expansion and multi-passaging of the neurospheres may be a change in the developmental properties of the progenitors. As an example, the multipassing of midbrain neurospheres results in a complete loss of their ability to differentiate into a dopaminergic neurotransmitter phenotype.

In the present study, we have characterized primary neurospheres from mouse ventral rostral hindbrain at embryonic day 12 (E12). The generated neurospheres were regionally specified and comprised a mixed cell population including proliferative active multipotent hindbrain progenitors whose differentiation could be triggered toward the 5-HT phenotype. We therefore suggest that this culture system is a useful tool for studying the molecular mechanisms underlying 5-HT development.

\section{Materials and methods}

Recombinant human (rh) N-terminal Shh was kindly provided by Dr. A. Galdes (Biogen, Cambridge, Mass.). FGF2 and epidermal growth factor (EGF) were obtained from Invitrogen (Karlsruhe, Germany). Lyophylized factors were resuspended in Dulbecco's modified Eagle's medium (DMEM; Gibco) with $0.25 \%$ bovine serum albumin (BSA; cell-culture grade; Sigma), $100 \mathrm{U} / \mathrm{ml}$ penicillin, $0.5 \mu \mathrm{g} / \mathrm{ml}$ streptomycin, $100 \mu \mathrm{g} / \mathrm{ml}$ neomycin (all from Gibco) to give a final factor concentration of $1 \mu \mathrm{g} / \mathrm{ml}$ and were stored in aliquots of $50-100 \mu 1$ at $-70^{\circ} \mathrm{C}$ until use. The function-blocking anti-Shh antibody was obtained from Developmental Studies Hybridoma Bank (Iowa City, Iowa).

Primary neurosphere culture of mouse ventral rostral hindbrain at E12

Pregnant Naval Medical Research Institute (NMRI) mice were sacrificed by cervical dislocation, and E12 embryos were collected in $\mathrm{Ca}^{2+}-\mathrm{Mg}^{2+}$-free Hanks' balanced salt solution (Sigma-Aldrich, Schnelldorf, Germany). Experiments were performed according to the National Institutes of Health and ethical regulations. The day of vaginal plug identification was designated as E1. The meninges were excised, and the ventral rostral hindbrain was dissected and incubated in $0.15 \%$ trypsin/EDTA (Gibco BRL, Eggenstein, Germany) for $15 \mathrm{~min}$ at $37^{\circ} \mathrm{C}$. Small tissue pieces were further dissociated mechanically by gentle trituration in fire-polished siliconized Pasteur pipettes. Dissociated cells were resuspended in high glucose Dulbecco's modified Eagle's medium (DMEM-F12) supplemented with $0.25 \%$ BSA, N1 additives, $100 \mathrm{U} / \mathrm{ml}$ penicillin, $0.5 \mu \mathrm{g} / \mathrm{ml}$ streptomycin, and $100 \mu \mathrm{g} / \mathrm{ml}$ neomycin (Gibco BRL). Cells were cultured in serum-free medium on non-coated culture dishes at a density of $5 \times 10^{5}$ cells $/ 3 \mathrm{ml}$ per dish in the presence of FGF2 $(20 \mathrm{ng} / \mathrm{ml})$ and EGF $(20 \mathrm{ng} / \mathrm{ml})$. The formation of neurospheres was evident after a culture time of approximately 4 days. The neurospheres were allowed to expand for 10 days. Primary neurospheres from mouse E12 ventral midbrain were essentially generated as previously described (Roussa et al. 2006). Subsequently, ventral midbrain and ventral hindbrain neurospheres were either processed for RNA isolation and gene expression analysis or were dissociated as described below. Total mRNA was isolated according to the manufacturer's protocol (Rneasy, 
Qiagen, Hilden, Germany). For immunocytochemical analysis, neurospheres were enzymatically and mechanically dissociated and plated at a density of $2 \times 10^{5}$ cells onto polyornithine (PORN)- and laminin-coated (Sigma-Aldrich) $12-\mathrm{mm}^{2}$ glass cover-slips. Cells were incubated in serumfree medium in a $95 \%$ air $/ 5 \% \mathrm{CO}_{2}$ atmosphere at $37^{\circ} \mathrm{C}$. One day after plating (day in vitro [DIV]1), factors were applied at a final volume of $750 \mu 1$ medium at the following concentrations: Shh $(1 \mathrm{nM})$ or anti-Shh $(2.5 \mu \mathrm{g} / \mathrm{ml})$. At DIV3, cells were fixed in 4\% paraformaldehyde (PFA) and processed for immunocytochemistry.

\section{Immunocytochemistry}

PFA-fixed cells were permeabilized with acetone for $10 \mathrm{~min}$ at $-20^{\circ} \mathrm{C}$ and blocked with $10 \%$ normal goat serum (NGS, Dako, Hamburg, Germany) in phosphate-buffered saline (PBS). Subsequently, cells were incubated with primary antibodies anti-glial fibrillary acidic protein (GFAP; rabbit polyclonal, 1:1,000; Dako, Hamburg, Germany), anti-nestin (1:20), and anti- $\beta$ III tubulin (mouse monoclonal, 1:20; Developmental Studies Hybridoma Bank, University of Iowa, USA), or anti-O4 (mouse monoclonal, 1:400; Chemicon/Millipore, Hofheim, Germany) diluted in PBS containing $10 \%$ NGS and $0.1 \%$ Triton X-100 overnight at $4^{\circ} \mathrm{C}$. Cells were washed with PBS and incubated with indocarbocyanin (CY3) or fluorescein isothiocyanate (FITC)-conjugated donkey-anti-rabbit or donkey-anti-mouse IgGs as secondary antibodies (Jackson Immuno Research Europe, Newmarket, Suffolk, UK) for $1 \mathrm{~h}$ at room temperature. Controls were carried out by omitting the primary antibody. For analysis of 5-HT immunoreactivity, cells were fixed in methanol/acetone (1:1) for $10 \mathrm{~min}$ on ice and washed with PBS. A blocking step with $10 \%$ NGS in PBS was followed by incubation with anti-5-HT antibody (rabbit polyclonal, 1:1,000; Sigma-Aldrich) in PBS containing 10\% NGS and $0.1 \%$ Triton X-100 overnight at $4^{\circ} \mathrm{C}$. After being washed with PBS, cells were exposed to a biotinylated anti-rabbit (1:200; Sigma) antibody for $1 \mathrm{~h}$ at room temperature, washed with PBS, and finally incubated with streptavidin-FITC (1:200; Dako) for $30 \mathrm{~min}$ at room temperature. Cell nuclei were stained with 4'-,6'-diamidino-2-phenylindole dihydrochloride (DAPI; Sigma-Aldrich; $1 \mathrm{mg} / \mathrm{ml}$ stock solution) diluted 1:1,000 in PBS for 5 min. Cells were washed with PBS, mounted with Vectashield (Vector Laboratories, Peterborough, UK), and viewed with a fluorescence microscope (Zeiss, Jena, Deutschland).

Reverse transcription with polymerase chain reaction

Total RNA was isolated from tissue dissected from mouse rostral ventral hindbrain (E12) and from primary neurospheres derived from the same brain region by using the
Qiagen Rneasy kit (Qiagen, Hilden, Germany). Reverse transcription (RT) was carried out on $1 \mu \mathrm{g}$ RNA in $20-\mu 1$ reactions containing $1 \mu 1$ oligo(dT)primers (Invitrogen, Karlsruhe, Germany) and $2 \mu 1 \quad 10 \mathrm{mM}$ dNTP mix (Eppendorf, Hamburg, Germany). After denaturation for $10 \mathrm{~min}$ at $70^{\circ} \mathrm{C}$ and annealing on ice, $10 \times$ RT buffer, $1 \mathrm{U}$ M-MLV reverse transcriptase, and $10 \mathrm{U}$ RNAse inhibitor (Fermentas, St. Leon-Roth, Germany) were added. The reaction was performed for $60 \mathrm{~min}$ at $42^{\circ} \mathrm{C}$, followed by a terminal denaturation for $10 \mathrm{~min}$ at $95^{\circ} \mathrm{C}$. Parallel reactions without M-MLV reverse transcriptase were used as negative controls. The forward (F) and reverse (R) primers used for gene expression analysis are shown in Table 1.

The polymerase chain reaction (PCR) was performed in $30-\mu 1$ reactions containing $0.2 \mu \mathrm{M}$ specific primers, $200 \mu \mathrm{M}$ deoxynucleoside-triphosphates (Eppendorf), 10× reaction buffer, $2 \mathrm{mM} \mathrm{MgCl}_{2}, 1 \mathrm{U}$ Taq polymerase (Fermentas), and $1 \mu \mathrm{l}$ of the RT reaction as template. Amplification was carried out under the following conditions: initial denaturation for $10 \mathrm{~min}$ at $95^{\circ} \mathrm{C}$, denaturation for $1 \mathrm{~min}$ at $94^{\circ} \mathrm{C}$, primer annealing for $40 \mathrm{~s}$ (at the appropriate temperature for each primer pair), and elongation for $1 \mathrm{~min}$ at $72^{\circ} \mathrm{C}$. After 35 cycles, the amplification was terminated with a final extention for $10 \mathrm{~min}$ at $72^{\circ} \mathrm{C}$, followed by rapid cooling to $4^{\circ} \mathrm{C}$. Amplification products were separated by agarose gel electrophoresis and visualized by ethidium-bromide staining.

\section{5'-Bromodeoxyuridine incorporation assay}

Cell proliferation was monitored by the 5 '-bromodeoxyuridine (BrdU) incorporation assay. BrdU (Boehringer, Ingelheim, Germany) was added to the primary neurosphere culture medium for $2,4,6$, or $8 \mathrm{~h}$ at a final concentration of $10 \mu \mathrm{M}$. Primary neurospheres were subsequently plated on PORN/ laminin-coated $12-\mathrm{mm}^{2}$ glass cover-slips. Cells were fixed at DIV3 with $70 \%$ ethanol (in $50 \mathrm{mM}$ glycine buffer, $\mathrm{pH} 2$ ) for $20 \mathrm{~min}$. After multiple washing steps, cells were incubated with anti-BrdU mouse monoclonal antibody (1:10; Boehringer). Anti-mouse IgG coupled to FITC was used as the secondary antibody. BrdU-positive cells expressing $\beta$-III-tubulin or $\mathrm{O} 4$ were detected by doubleimmunostaining with the respective specific antibodies as second primary antibodies.

\section{Statistics}

Data are presented as the mean \pm standard error of mean (SEM). Statistical analysis was performed by using the Student's double $t$-test. Differences were considered statistically significant at $* P<0.05, * * P<0.01$, and $* * * P<0.001$. 
Table 1 Primer sequences used for gene expression analysis by reverse transcription with polymerase chain reaction $(P C R)$

\begin{tabular}{|c|c|c|c|}
\hline Gene & Primer & Genbank accession number & $\begin{array}{l}\text { Amplified PCR fragment } \\
\text { (nucleotide position) }\end{array}$ \\
\hline Gapdh F & 5'-AGTATGATGACATCAAGAAGG-3' & NM008084 & $804-1210$ \\
\hline Gapdh $\mathrm{R}$ & 5'-ATGGTATTCAAGAGAGTAGGG-3' & & \\
\hline Nestin F & 5'-CAG GCT TCT CTT GGC TTT CCT G-3' & NM016701 & $1074-1512$ \\
\hline Nestin R & 5'-GGT GAG GGT TGA GGG GTG G-3' & & \\
\hline$\beta$-III-Tubulin $\mathrm{F}$ & 5'-ACCTTGTGTCTGCCACCATGA-3' & NM023279 & $722-1049$ \\
\hline$\beta$-III-Tubulin $\mathrm{R}$ & 5'-TTACTCTGGATGGCCAGCAT-3' & & \\
\hline$G b \times 2 \mathrm{~F}$ & 5'-TCTGGAGAGCGATGTGGATTA-3' & NM010262 & $1021-1428$ \\
\hline$G b x 2 \mathrm{R}$ & 5'-ATAGCGAACCTGCTAACGTGA-3' & & \\
\hline Otx2 F & 5'-TATGGAGCTTCAAGCTTGGGT-3' & NM144841 & $1059-1393$ \\
\hline Otx2 R & 5'-GTCCATTTCAGGTTGCTGGTT-3' & & \\
\hline $\operatorname{Shh} \mathrm{F}$ & 5'-TGATGTGTGGGCCCGGCAGGGGGTTT-3' & NM009170 & $411-934$ \\
\hline $\operatorname{Shh} \mathrm{R}$ & 5'-TCAGCCGCCGGATTTGGCCGCCACG-3' & & \\
\hline Fgf4 F & 5'-TCTACTGCAACGTGGGCAT-3' & NM010202 & $364-719$ \\
\hline Fgf4 R & 5'-AGGAAGGAAGTGGGTTACCTT-3' & & \\
\hline$F g f 8 \mathrm{~F}$ & 5'-GTAGTTGAGGAACTCGAAGCG-3' & AF520763 & $181-675$ \\
\hline$F g f 8 R$ & 5'-TTTACACAGCATGTGAGGGAG-3' & & \\
\hline$N k x 2.2 \mathrm{~F}$ & 5'-GAAGCGCCGAGTGCTCTTCTCC-3' & AY410225 & $108-482$ \\
\hline$N k x 2.2 \mathrm{R}$ & 5'-GCCGAGCTGTACTGGGCGTTGT-3' & & \\
\hline $\operatorname{Lmx} 1 b \mathrm{~F}$ & 5'-ATGTTGGACGGCATCAAGAT-3' & NM010725 & $1-493$ \\
\hline $\operatorname{Lmx} 1 b \mathrm{R}$ & 5'-TCACAGAGTCAGACTCGTCCG-3' & & \\
\hline Gata $2 \mathrm{~F}$ & 5'-CACCCCGCCGTATTGAATG-3' & NM008090 & $295-424$ \\
\hline Gata2 R & 5'-CCTGCGAGTCGAGATGGTTG-3' & & \\
\hline Pet1 F & 5'-AGCAAGCCTAACATGAACTACG-3' & NM153111 & $484-593$ \\
\hline Pet1 R & 5'-AAGTCAAAGCGGTAGGCG-3' & & \\
\hline$T p h 2 \mathrm{~F}$ & 5'-AAGTCGAAATCTTCGTGGACTG-3' & NM173391 & $432-531$ \\
\hline Tph2 R & 5'-GGCGGATTCAGGGTCACAAT-3' & & \\
\hline Gfap F & 5'-AGGCAGAGAACAACCTGGCT-3' & NM01077 & $496-593$ \\
\hline Gfap R & 5'-CTCCTCCTCCAGCGATTCAA-3' & & \\
\hline
\end{tabular}

\section{Results}

Primary neurospheres of mouse E12 ventral rostral hindbrain contain cells with proliferative capacity

Although neurospheres formation has been documented from various regions of the embryonic and adult brain (Reynolds and Weiss 1992; Morshead et al. 1994; Palmer et al. 1997; Gage 2000; Roisen et al. 2001; Roussa et al. 2006), data related to the hindbrain are lacking. We have therefore investigated whether cells derived from mouse rostral hindbrain at E12 also have the capacity for neurosphere formation. Free-floating cell aggregates generated by dissociated cells of mouse E12 ventral rostral hindbrain and cultured under serum-free conditions in the presence of EGF and FGF2 are illustrated in Fig. 1a, b. Neurospheres so formed were detectable after a culture time of approximately 4 days. Occasionally, some adhering neurospheres were also evident (Fig. 1a, arrow). Only single non-viable cells could be observed (shown at higher magnification in Fig. 1b, asterix).

In order to establish an adequate in vitro system that could mimic the in vivo situation, we first determined whether primary neurospheres contained cells that had not yet exited the cell cycle. The proliferation activity of the cells within the primary neurospheres was first analyzed by BrdU incorporation experiments. Neurospheres were treated with BrdU for various time periods before plating. As shown in Fig. 2a-c, BrdU was detected after incubation for $2 \mathrm{~h}$ (Fig. 2a), 4 h (Fig. 2b), and 6 h (Fig. 2c). At all time points examined, a high number of cells incorporated BrdU (green), indicative of cell division. BrdU-negative cells could also be observed.

The presence of progenitor cells within the generated mouse E12 rostral hindbrain primary neurospheres was determined by immunocytochemistry for nestin, an established marker for neural and glial precursors. After dissociation of the neurospheres and plating, a large number of nestin-positive cells were present in the cultures (Fig. 2d).

Cellular composition of primary neurospheres from mouse E12 ventral rostral hindbrain

The cellular composition of generated primary neurospheres was analyzed by RT-PCR. The gene expression pattern of 


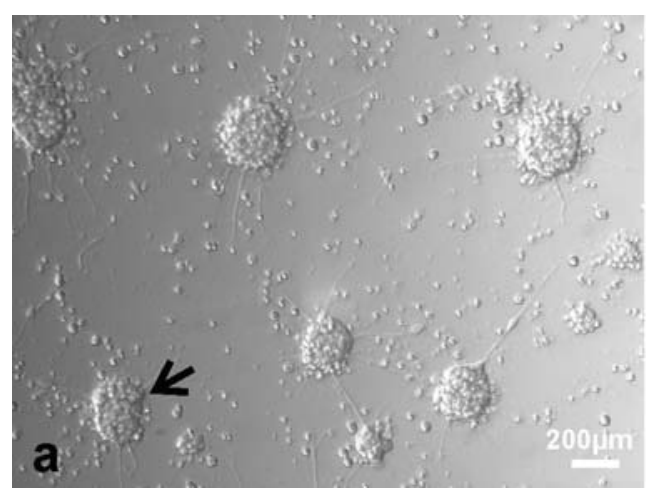

Fig. 1 Cells derived from mouse embryonic day 12 (E12) rostral hindbrain may form neurospheres. a Free-floating primary neurospheres from mouse ventral rostral hindbrain formed in response to FGF2 $(20 \mathrm{ng} / \mathrm{ml})$ and EGF $(20 \mathrm{ng} / \mathrm{ml})$ under serum-free conditions.

primary neurospheres from ventral hindbrain was compared with that of primary tissue of the same brain region (Fig. 3a-d, NS and T, respectively). Expression of Otx2, a gene exclusively expressed in the midbrain (Millet et al. 1999), was neither detectable in dissected tissue from mouse E12 ventral rostral hindbrain (Fig. 3a, lane 5), nor in primary neurospheres (Fig. 3a, lane 4). In contrast, Gbx2 expression, a determinant of the hindbrain region (Wassarman et al. 1997), was clearly evident in primary tissue (Fig. 3a, lane 7) and in primary neurospheres (Fig. 3a, lane 6). Nestin, a marker for progenitor cells (Lendahl et al. 1990), could be detected in both primary tissue (Fig. 3b, lane 3 ) and primary

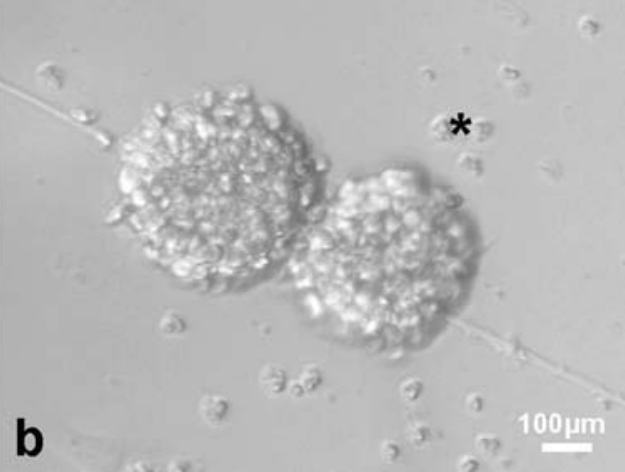

Occasionally, single adhering neurospheres were detectable (arrow). b Primary neurospheres at higher magnification. Non-viable cells (asterisk) were rarely present in the cultures

neurospheres (Fig. 3b, lane 2) generated from the same brain region. Expression of the neuron-specific marker $\beta$-III tubulin (Caccamo et al. 1989) could also be determined in both samples (Fig. 3b, lanes 4 - 5), although its expression was considerably higher in primary tissue than in neurospheres. In contrast to $\beta$-III tubulin, Gfap, a general glial cell marker (Eng et al. 2002), was detectable in primary tissue (Fig. 3a, lane 10) but not in primary neurospheres of ventral rostral hindbrain (Fig. 3a, lane 9).

The endogenous expression of molecules involved in the patterning of the neural tube and relevant as early determinants of 5-HT neuron development, namely Shh,
Fig. 2 a Primary neurospheres from mouse E12 rostral hindbrain exhibit proliferation activity. a-c BrdU incorporation analysis of primary neurospheres generated from mouse rostral ventral hindbrain (E12) Neurospheres were treated with BrdU $(10 \mu \mathrm{M}$ final concentration) for $2 \mathrm{~h}, 4 \mathrm{~h}$, and $6 \mathrm{~h}$ and subsequently plated. BrdU was visualized by immunocytochemistry (green). d Dissociated primary neurospheres at day in vitro 3 (DIV3) from ventral rostral hindbrain E12 immunostained for nestin (red), a marker for neural stem/progenitor cells. Nuclei were counterstained with DAPI (blue)
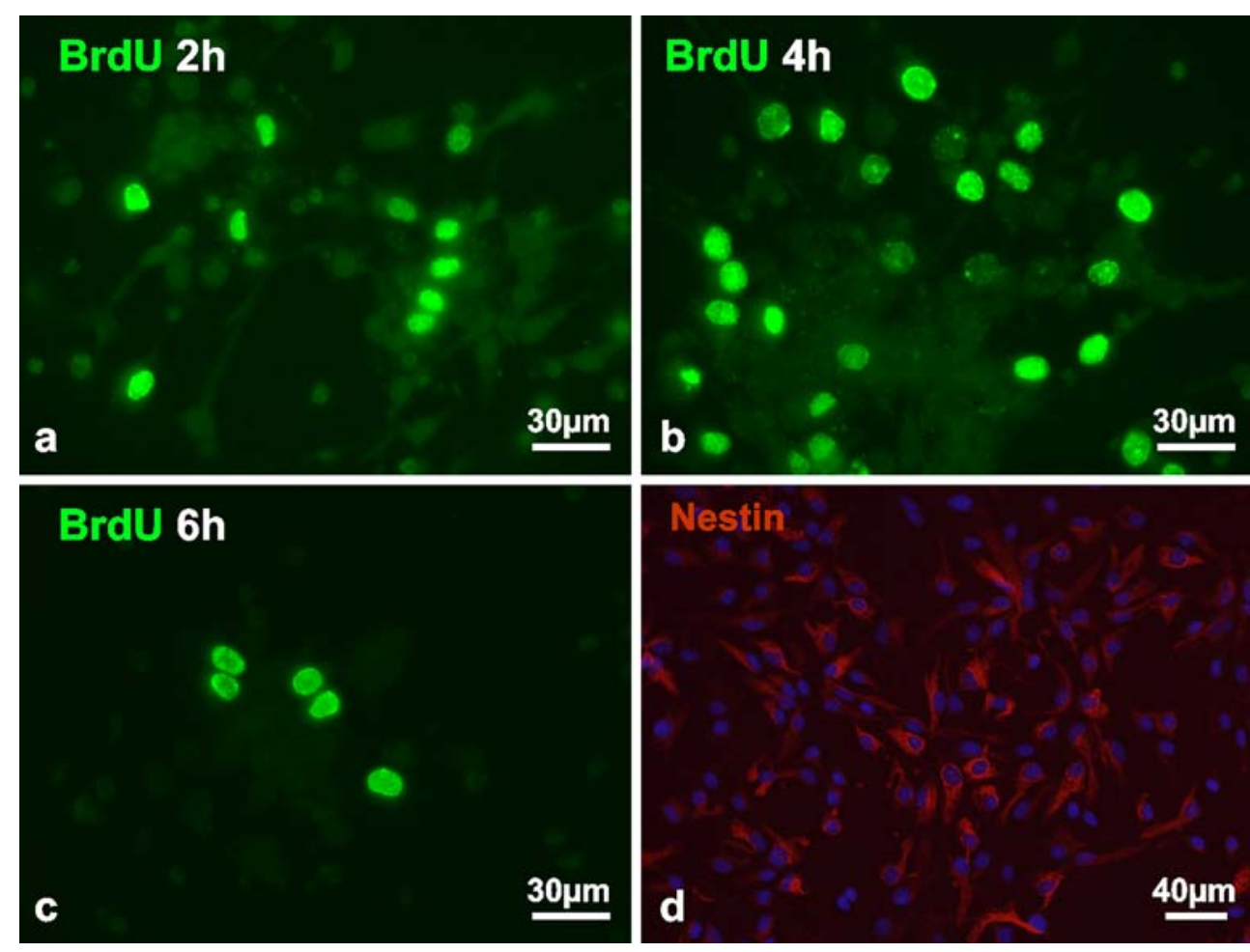
Fig. 3 Comparison of gene expression pattern of primary neurospheres (NS) and primary tissue (T) from mouse E12 ventral rostral hindbrain by using RT-PCR. a, b Expression of Otx 2 and Gbx2, determinants of midbrain and hindbrain, respectively, demonstrates successful tissue dissection. Expression of Gfap, Nestin, and $\beta$-III tubulin (markers of glia, precursors, and neurons, respectively) indicates a mixed cellular population within the primary neurospheres. c Determination of the endogenous expression of key signaling molecules involved in the patterning of neural tube (Shh, Fgf4, and Fgf8) and of early determinants of

5-HT neuron development $(N k x 2.2, L m x 1 b)$ in primary neurospheres (NS) compared with primary tissue $(\mathrm{T})$ of mouse E12 ventral rostral hindbrain. d Expression analysis of the early 5-HT precursor marker gene Gata2 and genes required for terminal 5-HT phenotype specification, viz., Pet1 and Tph2, in primary neurospheres (NS) and primary tissue (T) of mouse E12 ventral rostral hindbrain

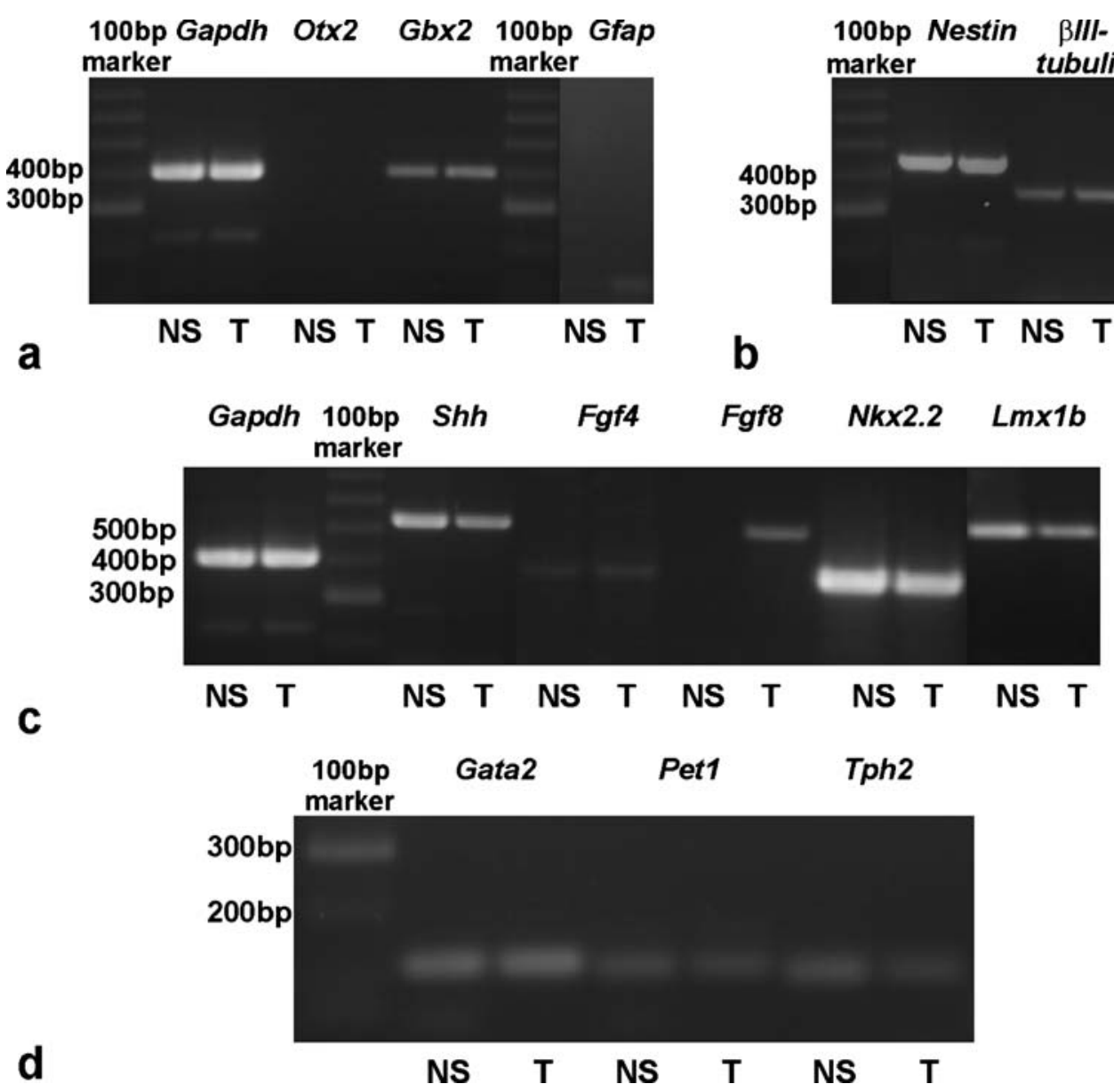

phenotype (Goridis and Rohrer 2002), was found to be markedly stronger in primary neurospheres (Fig. 3d, lanes 4, 6) than in the respective primary tissue (Fig. 3d, lanes 5, 7).

Differentiation capacity of multipotent progenitors within primary neurospheres of mouse ventral rostral hindbrain at E12

The cellular composition of the generated primary neurospheres was further tested by immunocytochemistry with specific markers for neural cell lineages. Primary neurospheres were dissociated into single cells, plated onto PORN/ laminincoated cover-slips and allowed to differentiate. As expected from this time point of differentiation, immunoreactivity for GFAP, a marker for glial cells was weak or absent (Fig. 4a). In contrast, at 3 days after plating (Fig. 4b), a considerable number of cells had differentiated into neurons, as indicated by $\beta$-III tubulin staining. In addition, cells revealing 5-HT immunoreactivity could also be found (Fig. 4c). When primary neurospheres from mouse E12 ventral midbrain were dissociated and stained for 5-HT, no specific labeling could be observed (Fig. 4d). Multipotency of progenitors within the generated primary rostral hindbrain neurospheres was inves- 
Fig. 4 Dissociated primary neurospheres of mouse E12 ventral rostral hindbrain reveal a mixed cellular composition as shown by immunostaining with markers for astrocytes (a, GFAP, red) and neurons (b, $\beta$-III tubulin, green). Many generated neurons were 5-HT positive (c, green). Primary neurospheres derived from mouse ventral midbrain were devoid of 5-HT immunoreactivity (d). Nuclei in a-d were counterstained with DAPI (blue). Many $\beta$ -

III-tubulin cells were also immunoreactive for BrdU; the mature neuronal morphology of the generated neurons is revealed at higher magnification (e). Double immunofluorescence (f) for BrdU (green) and the oligodendrocytes marker $\mathrm{O} 4$ (red) showed that BrdU and $\mathrm{O} 4$ were co-localized in some, but not all cells
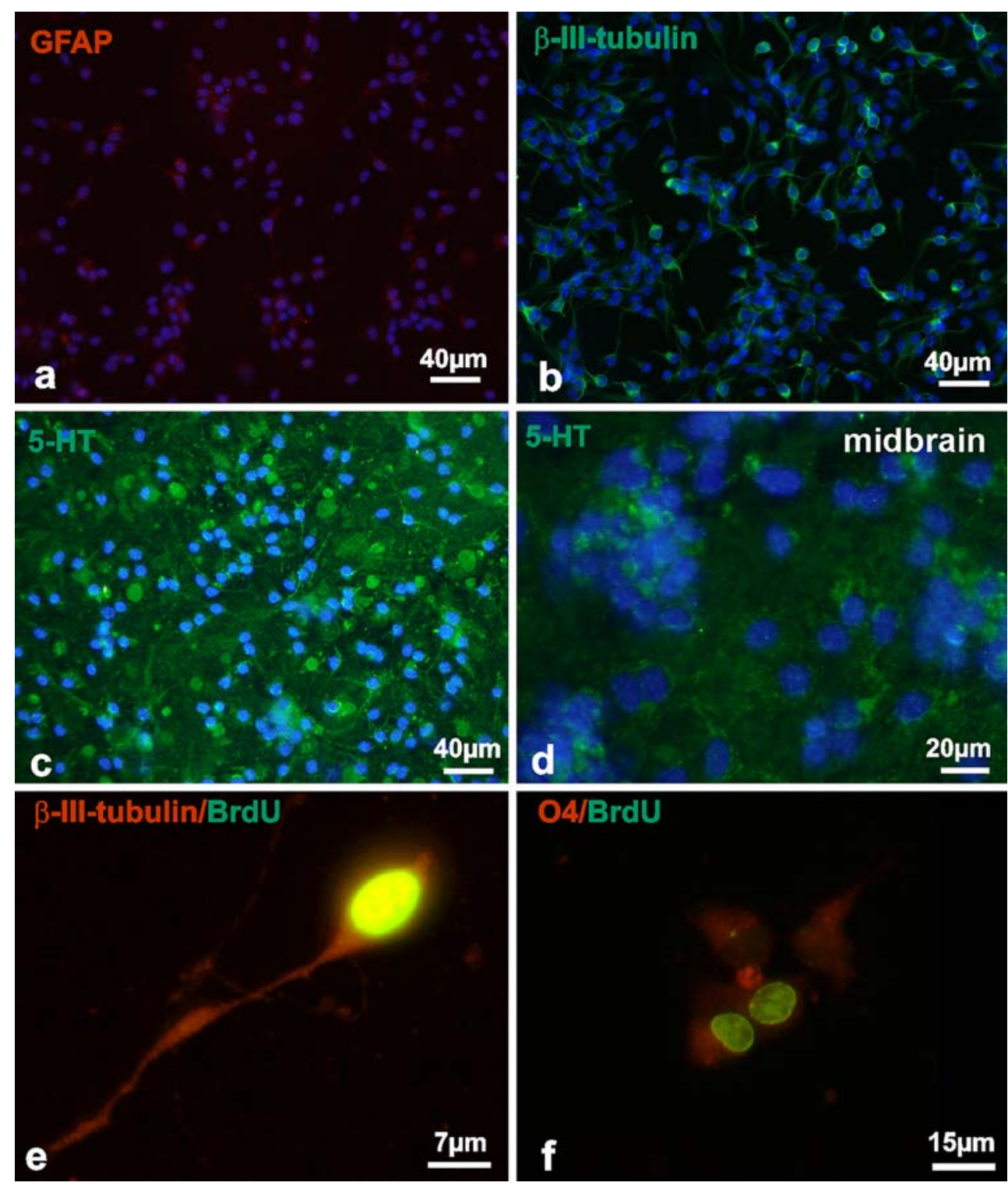

tigated by double immunofluorescence for BrdU/neural lineage marker. As shown in Fig. 4e, $\beta$-III-tubulin cells were additionally positive for BrdU and showed a mature neuronal morphology, indicating that these neurons were derived from proliferating precursor cells within the neurospheres. Finally, O4-positive cells could be detected (Fig. 4f) that were either positive or negative for BrdU. Taken together, these data demonstrate the mixed cell population in the primary neurospheres and, at the same time, the presence of multipotent hindbrain progenitors that lie within the neurospheres and that can differentiate toward neural lineages.

Shh is required for differentiation of 5-HT neurons in vitro

The signaling molecule Shh is an established key player for the development of ventral neuronal populations, including
5-HT neurons in vivo. Treatment of dissociated neurospheres from mouse E12 ventral rostral hinbrain with exogenous Shh significantly increased the number of 5-HT-immunoreactive cells, compared with the untreated controls (Fig. 5b, d; $* P=0.04, n=5)$. In addition, neutralization of endogenous Shh (Fig. 5c) by using an anti-Shh function blocking antibody significantly reduced the number of 5-HT-positive neurons compared with the control cultures (Fig. 5d; $* * P=0.006 ; n=5)$.

Figure 5e illustrates 5-HT immunoreactivity in dissociated neurospheres derived from mouse E12 ventral midbrain. Other than unspecific background staining, no 5-HT-positive neurons could be detected. Moreover, after treatment of dissociated neurospheres with exogenous Shh, no changes in the number of 5-HT-positive cells could be observed, indicating that the effect of Shh on dissociated neurospheres 
Fig. $5 \mathrm{Shh}$ is necessary for differentiation of 5-HT neurons in vitro. Differentiation potential of neurospheres derived from mouse E12 ventral hindbrain after factor treatment. Shh treatment of the cultures significantly increased the number of 5-HT positive neurons $(\mathbf{b}, \mathbf{d})$ compared with untreated controls (a, d). Neutralization of endogenous Shh significantly decreased the number of 5HT-immunopositive neurons (c, d) compared with controls $(c t l)$. Data are given as mean \pm SEM ( $n=5), P$-values derived from Student's t-test are $* P<0.05$ for increased numbers of immunoreactive cells after factor treatment as compared with untreated controls, and ${ }^{++} P<0.01$ for decreased numbers of immunoreactive cells after factor treatment as compared with untreated controls. e, f Treatment of dissociated primary neurospheres derived from ventral midbrain did not induce 5-HT immunoreactivity
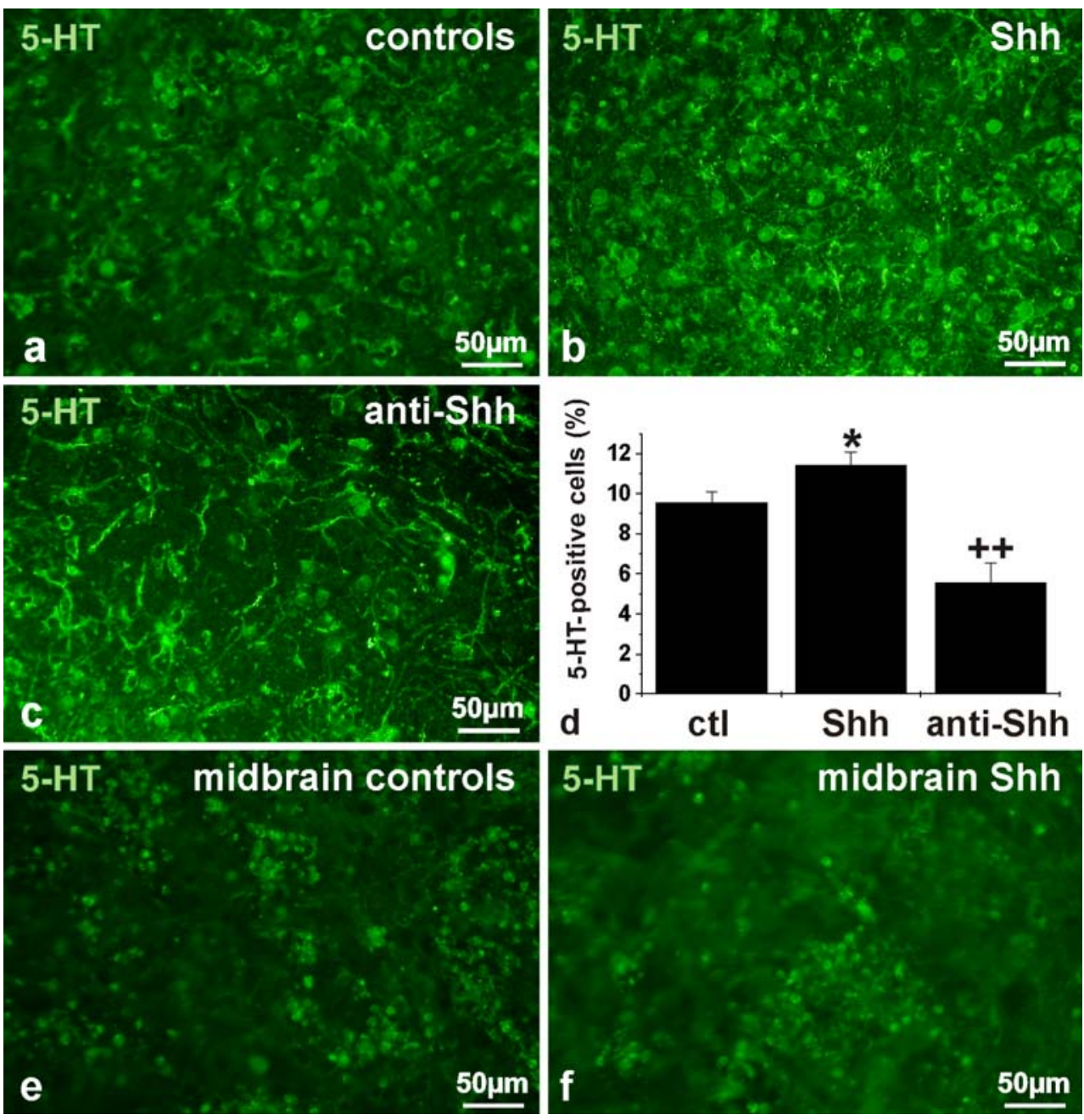

from ventral hindbrain was specific for this brain area. Further, these data demonstrate that the generated culture system is suitable for studying the development of 5-HT neurons in vitro.

\section{Discussion}

To elucidate the complex network of intrinsic and extrinsic molecules directing the differentiation of hindbrain progenitor cells toward 5-HT neurotransmitter phenotype, a wellcharacterized in vitro system is required that mimics biology as closely as possible. In the present study, we have established a primary neurosphere culture system from mouse ventral rostral hindbrain at E12. Ventral rostral hindbrain tissue has been dissociated and cultured under serum-free conditions in the presence of the mitogens FGF2 and EGF. The formation of neurospheres has been observed within a few days. Whereas a number of studies dealing with the isolation of neurospheres from various areas of the CNS give the impression that this culture system is well documented, nothing could be further from the truth (Reynolds and Weiss 1992; Morshead et al. 1994; Palmer et al. 1997; Gage 2000; Roisen et al. 2001; Roussa et al. 2006). Neurospheres, i.e., free-floating cell aggregates, have been considered to allow the propagation of progenitor cells and additionally enable the experimental control, monitoring, and triggering of neural precursors toward a neuronal fate and neurotransmitter phenotype. However, the term "neurospheres" has been used for considerably different experimental approaches, such as clonal neurospheres, adherent neurospheres, and multi-passaged neurospheres. In order to characterize our culture system and to test it for its suitability in studies of the differentiation of 5-HT neurons in vitro, the following questions have been addressed: (1) what is the cellular composition of this neurosphere culture system; (2) what are the advantages and limitations of this culture system?

An investigation of the gene expression in the primary neurospheres and in dissected ventral hindbrain primary tissue has been performed by RT-PCR. The purity of dissected ventral rostral hindbrain, by means of exact tissue dissection, has been tested by an analysis for Otx2 and Gbx2 expression 
(Fig. 3a), determinants of the midbrain-hindbrain boundary (Millet et al. 1999; Brodski et al. 2003). The absence of Otx2 expression in ventral hindbrain tissue and primary neurospheres excludes contamination with dissected midbrain tissue. In addition, the generated primary neurospheres retain their regional specification, as indicated by the high expression of $G b x 2$, a determinant for the hindbrain (Wassarman et al. 1997), by the endogenous expression of the established key signaling molecules for 5-HT development Shh and Fgf4 (Ye et al. 1998), and by the expression of the serotonergic lineage-specific transcription factors Gata2 and Pet1 (Goridis and Rohrer 2002). Regional specification of primary neurospheres has been observed in the midbrain, cortex, and striatum (Hitoshi et al. 2002; Ostenfeld et al. 2002; Parmar et al. 2002; Roussa et al. 2006; Armando et al. 2007). Interestingly, Fgf8 expression has been detected in primary hindbrain tissue but not in primary neurospheres. This result can be explained by the absence of the isthmus, which induces $\mathrm{Fg} 8 \mathrm{8}$ expression.

The generated primary neurospheres comprise a mixed cell population, including multipotent hindbrain progenitors, postmitotic differentiated cells, and fully differentiated 5-HT neurons. Several lines of evidence suggest that the generated neurospheres contain cells that proliferate actively; many cells within the neurospheres have revealed a protein abundance for nestin (Fig. 2d) or are positive for $\mathrm{BrdU}$, indicative of proliferating hindbrain progenitors. The data also suggest that the formation of the free-floating cell clusters is indeed caused by the proliferation of the hindbrain precursor cells rather than being based on cell re-aggregation. The presence of cells committed to the neuronal and glial lineages has been determined by immunocytochemistry for the markers $\beta$-III tubulin and O4, respectively. Several cells within the neurospheres even enter the stage of final serotonergic phenotype specification, as shown by 5-HT immunofluorescence. These data have further been confirmed by RT-PCR analysis, as illustrated in Fig. 3a-d. The observation that the expression of $N k x 2.2$, Pet1, and Lmxlb is higher in primary neurospheres, compared with primary hindbrain tissue, argues for an enrichment of hindbrain progenitors in the neurospheres and for the regional specificity of the culture. These progenitor cells may differentiate into the neuronal or glial lineage. Indeed, several cells show double-labeling for both BrdU and $\beta$-III tubulin (Fig. 4e) or for BrdU and $\mathrm{O} 4$ (Fig. 4f), clearly demonstrating that at least some of the neurons and oligodendrocytes identified in dissociated neurospheres are derived from precursor cells, rather than from differentiated cells within the neurospheres. At the same time, BrdU-negative but either $\beta$-III-tubulin-positive or O4-positive cells have also been observed, indicating that some cells within the neurospheres are committed to a specific cell fate before BrdU treatment. The presence of cells in different developmental stages within the culture system imitates the regional cellular composition in vivo and is therefore the system of choice for the investigation of the triggering of precursor cells toward a specific cell fate. Of course, this culture system is not suitable for studies focusing on the differentiation potential of neural stem cells in general. For the latter, clonal analysis is indispensable.

5-HT neurons reveal a close ontogenetic relationship to midbrain dopaminergic (DA) neurons. Both cell populations derive from ventral neuroepithelial progenitors located on either side of the midbrain-hindbrain boundary (Millet et al. 1999; Joyner et al. 2000; Brodski et al. 2003). Among the signaling molecules involved in the induction and specification of neuronal populations along the dorso-ventral axis of the developing brain, Shh has been identified as dictating the differentiation of both midbrain and hindbrain progenitors toward the respective neurotransmitter phenotype. The results of the present study have revealed significant increases in the number of 5-HT neurons after exogenous Shh treatment of dissociated mouse E12 primary neurospheres from rostral hindbrain but not from ventral midbrain. Accordingly, the neutralization of endogenous Shh significantly decreases the number of 5-HTimmunoreactive cells. These data demonstrate the specific responsiveness of the generated culture system to exogenous factors and indicate the experimental control of hindbrain progenitors toward the 5-HT phenotype.

We therefore conclude that the established primary neurosphere culture system is a useful model for in vitro studies aimed at elucidating the molecular mechanisms that direct the induction and specification of 5-HT neurons.

Open Access This article is distributed under the terms of the Creative Commons Attribution Noncommercial License which permits any noncommercial use, distribution, and reproduction in any medium, provided the original author(s) and source are credited.

\section{References}

Armando S, Lebrun A, Hugnot J-P, Ripoll C, Saunier M, Simonneau L (2007) Neurosphere-derived neural cells show region-specific behaviour in vitro. Dev Neurosci 18:1539-1542

Brodski C, Weisenhorn DM, Signore M, Sillaber I, Oesterheld M, Broccoli V, Acampora D, Simeone A, Wurst W (2003) Location and size of dopaminergic and serotonergic cell populations are controlled by the position of the midbrain-hindbrain organizer. J Neurosci 23:4199-4207

Caccamo D, Katsetos CD, Herman MM, Frankfurter A, Collins VP, Rubinstein LJ (1989) Immunohistochemistry of a spontaneous murine ovarian teratoma with neuroepithelial differentiation. Neuron-associated beta-tubulin as a marker for primitive neuropithelium. Lab Invest 60:390-398 
Chen ZF, Ding YQ (2006) Transcriptional control of the development of central serotonergic neurons. In: Thiel G (ed) Transcription factors in the nervous system. Wiley-VCH, Weinheim, pp 143-156

Cheng L, Chen CL, Luo P, Tan M, Qiu M, Johnson R, Ma Q (2003) Lmx1b, Pet-1, and Nkx2.2 coordinately specify serotonergic neurotransmitter phenotype. J Neurosci 23:9961-9967

Craven SE, Lim KC, Ye W, Engel JD, Sauvage F de, Rosenthal A (2004) Gata2 specifies serotonergic neurons downstream of sonic hedgehog. Development 131:1165-1173

Eng LF, Ghirnikar RS, Lee Y1 (2002) Glial fibrillary acidic protein: GFAP - thirty-one-years (1969-2000). Neurochem Res 25:14391451

Gage FH (2000) Mammalian neural stem cells. Science 287:1433-1438

Goridis C, Rohrer H (2002) Specification of catecholaminergic and serotonergic neurons. Nat Rev Neurosci 3:531-541

Hendricks T, Francis N, Fyodorov D, Deneris ES (1999) The ETS domain factor pet-1 is an early and precise marker of central serotonin neurons and interacts with a conserved element in serotonergic genes. J Neurosci 19:10348-10356

Hitoshi S, Tropepe V, Ekker M, Kooy D van der (2002) Neural stem cell lineages are regionally specified, but not committed, within distinct compartments of the developing brain. Development 129:233-244

Joyner AL, Liu A, Millet S (2000) Otx2, Gbx2 and Fgf8 interact to position and maintain a mid-hindbrain organizer. Curr Opin Cell Biol 12:736-741

Lendahl U, Zimmerman LB, McKay RD (1990) CNS stem cells express a new class of intermediate filament protein. Cell 60:585-595

Levitt P, Moore RY (1978) Developmental organization of raphe serotonin neuron groups in the rat. Anat Embryol 154:241-251

Millet S, Campbell K, Epstein DJ, Losos K, Harris E, Joyner AL (1999) A role for Gbx2 in repression of Otx2 and positioning the $\mathrm{mid} /$ hindbrain organizer. Nature 401:161-164

Morshead CM, Reynolds BA, Craig CG, McBurney MW, Staines WA, Morassutti D, Weiss S, Kooy D van der (1994) Neural stem cells in the adult mammalian forebrain: a relatively quiescent subpopulation of subependymal cells. Neuron 13:1071-1082

Nelson RJ, Chiavegatto S (2001) Molecular basis of aggression. Trends Neurosci 24:713-719

Ostenfeld T, Joly E, Tai Y-T, Peters A, Caldwell M, Jauniaux E, Svendsen CN (2002) Regional specification of rodent and human neurospheres. Brain Res Dev Brain Res 134:43-55
Palmer TD, Takahashi J, Gage FH (1997) The adult rat hippocampus contains primordial neural stem cells. Mol Cell Neurosci 8:389404

Parmar M, Skogh C, Björklund A, Campbell K (2002) Regional specification of neurosphere cultures derived from subregions of the embryonic telencephalon. Mol Cell Neurosci 21:645-656

Pattyn A, Vallstedt A, Dias JM, Samad OA, Krumlauf R, Rijili FM, Brunet JF, Ericson J (2003) Coordinated temporal and spatial control of motor neuron and serotonergic neuron generation from a common pool of CNS progenitors. Genes Dev 17:729-737

Pattyn A, Simplicio N, Doorninck JH van, Goridis C, Guillemot F, Brunet JF (2004) Ascl1/Mash1 is required for the development of central serotonergic neurons. Nat Neurosci 7:589-595

Reynolds BA, Weiss S (1992) Generation of neurons and astrocytes from isolated cells of the adult mammalian central nervous system. Science 255:1707-1710

Roisen FJ, Kluebere KM, Lu CL, Hatcher LM, Dozier A, Shields CB, Maguire S (2001) Adult human olfactory stem cells. Brain Res 890:11-22

Roussa E, Wiehle M, Dünker N, Becker-Katins S, Oehlke O, Krieglstein K (2006) TGF- $\beta$ is required for differentiation of mouse mesencephalic progenitors into dopaminergic neurons in vitro and in vivo. Ectopic induction in dorsal mesencephalon. Stem Cell 24:2120-2129

Wassarman KM, Lewandoski M, Campbell K, Joyner AL, Rubenstein JL, Martinez S, Martin GR (1997) Specification of the anterior hindbrain and establishment of a normal mid/hindbrain organizer is dependent on Gbx2 gene function. Development 124:29232934

Ye W, Shimamura K, Rubenstein JL, Hynes MA, Rosenthal A (1998) FGF and Shh signals control dopaminergic and serotonergic cell fate in the anterior neural plate. Cell 93:755-766

Zhang X, Gainetdinov RR, Beaulieu JM, Sotnikova TD, Burch LH, Williams RB, Schwartz DA, Krishnan KR, Caron MG (2005) Loss-of function mutation in tryptophan hydroxylase-2 identified in unipolar major depression. Neuron 45:11-16

Zhao Z-Q, Scott M, Chiechio S, Wang J-S, Renner KJ, Gereau RW IV, Johnson RL, Deneris ES, Chen Z-F (2006) Lmxlb is required for maintenance of central serotonergic neurons and mice lacking central serotonergic system exhibit normal locomotor activity. J Neurosci 26:12781-12788 